\title{
Comparação entre duas concentrações de glicerol para a criopreservação de sêmen de suçuarana (Puma concolor) ${ }^{1}$
}

\author{
Thyara de Deco-Souza ${ }^{2}$, Tarcizio A.R. de Paula ${ }^{2 *}$, Deiler Sampaio Costa ${ }^{3}$, Eduardo \\ Paulino da Costa ${ }^{2}$, João Bosco Gonçalves de Barros ${ }^{2}$, Gediendson Ribeiro \\ de Araujo ${ }^{2}$ e Moacir Carreta-Jr²
}

\begin{abstract}
Deco-Souza T., Paula T.A.R., Costa D.S., Costa E.P., Barros J.B.G., Araujo G.R. \& Carreta-Jr M. 2013. [Comparison between two glycerol concentrations to cryopreservation of semen of mountain lions (Puma concolor).] Comparação entre duas concentrações de glicerol para a criopreservação de sêmen de suçuarana (Puma concolor). Pesquisa Veterinária Brasileira 33(4):512-516. Setor de Patologia Veterinária, Faculdade de Veterinária, Universidade Federal do Rio Grande do Sul, Av. Bento Gonçalves 9090, Porto Alegre, RS 91540-000, Brazil. E-mail: tarcizio@ufv.br

The development of biotechnologies of reproduction is an important tool for the conservation of wild animals threatened with extinction. Assisted reproduction procedures in mountain lions, however, are scarce, especially those related to sperm cryopreservation. In this context, this study aimed to evaluate the freezing capacity of semen from adult mountain lions in captivity through the comparison of two concentrations of glycerol in the freezing media. Five adult male mountain lions were used, held at the Rehabilitation Center for Wild Animals of Mato Grosso do Sul (CRAS/MS). Samples were collected by electroejaculation and evaluated for physical appearance, volume, sperm progressive status, sperm motility, sperm concentration and sperm motility index. Each ejaculate was spliced into two aliquots and diluted in Tris-citrate-half egg yolk, at final concentrations of 5 and $7.5 \%$ glycerol, cooled at a rate of $-0.55^{\circ} \mathrm{C} / \mathrm{min}$ and frozen at a rate of $-5.8^{\circ} \mathrm{C} / \mathrm{min}$. Once thawed, the samples were re-evaluated and tested for thermoresistance and hypoosmotic swelling. The suggested protocol for cryopreservation and thawing of semen is efficient in both glycerol concentrations tested, with no difference $(p>0.05)$ between them.
\end{abstract}

INDEX TERMS: Mountain lion, Puma concolor, electroejaculation, semen, assistedreproduction, spermatic cell.

RESUMO.- 0 desenvolvimento de biotécnicas de reprodução é uma importante ferramenta para a conservação de animais silvestres ameaçados de extinção. Procedimentos de reprodução assistida em suçuarana, no entanto, são escassos na literatura, em especial aqueles relacionados à criopreservação de sêmen. Neste sentido, o presente trabalho objetivou avaliar a congelabilidade do sêmen de suçuaranas adultas mantidas em cativeiro, por meio da comparação entre duas

\footnotetext{
${ }^{1}$ Recebido em 31 de julho de 2012.

Aceito para publicação em 18 de setembro de 2012.

${ }^{2}$ Departamento de Veterinária, Universidade Federal de Viçosa (UFV), Av. P.H. Rolfs s/n, Centro, Viçosa, MG 36570-000, Brasil. *Autor para correspondência: tarcizio@ufv.br.

${ }^{3}$ Faculdade de Medicina Veterinária e Zootecnia, Universidade Federal do Mato Grosso do Sul (UFMS), Av. Felinto Miller s/n, Cidade Universitária, Campo Grande, MS 79070-900, Brasil. E-mails dos outros autores: thydeco@yahoo.com.br; deiler@hin.ufms.br; epcosta@ufv.br; boscoj@ pop.com.br; gediendson@gmail.com; moacircjr@yahoo.com.br
}

concentrações de glicerol no meio de congelamento. Foram usados cinco machos adultos de suçuarana, mantidos no Centro de Reabilitação de Animais Silvestres do Mato Grosso do Sul (CRAS/MS). As amostras foram coletadas por eletroejaculação e avaliadas quanto ao seu aspecto físico, volume, vigor, motilidade, concentração e índice espermático. De cada ejaculado duas alíquotas foram diluídas em meio Tris-citrato-gema de ovo, em concentrações finais de 5 e 7,5\% de glicerol, resfriadas a uma taxa de $-0,55^{\circ} \mathrm{C} / \mathrm{min}$ e congeladas a uma taxa de $-5,8^{\circ} \mathrm{C} / \mathrm{min}$. Depois de descongeladas, as amostras foram reavaliadas e submetidas aos testes de termorresistência e hiposmótico. 0 protocolo de criopreservação e descongelamento de sêmen proposto se mostrou eficiente em ambas as concentrações de glicerol testadas, não havendo diferença $(p>0,05)$ entre estas.

TERMOS DE INDEXAÇÃO: Onça parda, Puma concolor, eletroejaculação, reprodução assistida, sêmen, célula espermática. 


\section{INTRODUÇÃO}

As tecnologias de reprodução assistida como a criopreservação de gametas, a inseminação artificial, a fertilização in vitro e a transferência de embriões vêm sendo cada vez mais aplicadas (Swanson et al. 2002, Baudiet al. 2008), e englobam uma diversidade de áreas inter-relacionadas, incluindo biologia de gametas, embriologia, endocrinologia e criobiologia.

Alguns danos causados pela criopreservação de gametas podem ser evitados ou pelo menos minimizados pela diluição da amostra em meio adequado para criopreservação. 0 crioprotetor usado rotineiramente (glicerol) promove proteção às células das consequências da cristalização ao aumentar a fração de água não congelada no meio extracelular. Porém sabe-se que ele, em determinadas concentrações e temperaturas, apresenta efeito tóxico sobre o espermatozoide, embora esta toxicidade varie significativamente entre as espécies (Fahy 1986, England 1993, Nelson et al. 1999, Holt 2000, Santos et al. 2003, Pesch \& Bergmann 2006).

Para o uso efetivo das tecnologias de criopreservação de gametas nas espécies de felídeos o estudo e a propagação do conhecimento básico e de novas tecnologias são necessários, pois há variações espécie-específicas que precisam ser consideradas no desenvolvimento destes protocolos (Swanson \& Brown 2004). A criopreservação de gametas viáveis é fundamental para a criação de um banco de reserva genômica e importante para a manutenção do potencial reprodutivo no futuro. Descrições de procedimentos de reprodução assistida em suçuarana, no entanto, são escassos na literatura (Miller et al. 1990), em especial aqueles relacionados à criopreservação de sêmen. Além disso, também são raros os estudos com sêmen de suçuarana com as subespécies encontradas no Brasil (Deco et al. 2010).

Neste sentido, o presente experimento objetivou a avaliação da congelabilidade do sêmen de suçuaranas adultos mantidos em cativeiro, por meio da comparação de duas concentrações de glicerol no meio de congelamento.

\section{MATERIAL E MÉTODOS}

Foram usados cinco machos adultos de suçuarana (Puma concolor), mantidos em cativeiro no Centro de Reabilitação de Animais Silvestres do Mato Grosso do Sul (CRAS-MS). A presente experimentação foi submetida e aprovada pela Comissão de Ética do Departamento de Veterinária da Universidade Federal de Viçosa (Registro no 21/2008) e autorizada pelo IBAMA (Proc. no 145611 de 17.4.2008).

Foi realizada uma coleta em cada animal. Para obtenção do sêmen, os animais foram contidos quimicamente pelo uso de dardos anestésicos e mantidos sob anestesia. Usou-se como protocolo anestésico a associação, via intramuscular, de cloridrato de cetamina (10mg/kg, Dopalen ${ }^{\circledR}$, Vetbrands, SP, Brasil) e cloridrato de xilazina $\left(1,2 \mathrm{mg} / \mathrm{kg}\right.$, Anasedan ${ }^{\circledR}$, Vetbrands, SP, Brasil). Os animais tiveram seus parâmetros vitais aferidos e avaliados, por um médico veterinário, durante e após o procedimento. Doses complementares da associação anestésica foram utilizadas quando necessário, a critério do Médico veterinário responsável. Os animais foram monitorados até que conseguissem manter-se em estação, garantindo uma recuperação pós-anestésica segura.

Previamente à coleta de sêmen, foi feito o esvaziamento da bexiga com sonda uretral estéril e seringa de $10 \mathrm{~mL}$ e posterior lavagem da bexiga com solução fisiológica estéril, a fim de evitar a contaminação da amostra com urina.

O sêmen foi coletado com auxílio de um aparelho de eletroejaculação (Eletrogen SA200 ${ }^{\circledR}$, Santa Lydia/SP, Brasil), equipado com um transdutor retal de $2,5 \mathrm{~cm}$ de diâmetro com três eletrodos longitudinais. Para tal, foram aplicados no máximo 4 séries de 10 estímulos de $16 \mathrm{~V}$ com intervalos de um minuto entre as séries. Cada estímulo levou aproximadamente um segundo para ir de $0 \mathrm{~V}$ a $16 \mathrm{~V}$, permanecendo por dois a três segundos, seguido por um retorno abrupto a $0 \mathrm{~V}$, sendo o intervalo entre os estímulos de dois a três segundos (Deco et al. 2010). Para uma aferição mais precisa do volume do ejaculado, utilizou-se uma micropipeta de volume ajustável.

Após as coletas, os ejaculados foram analisados quanto aos aspectos físicos (volume e cor). Imediatamente após esta análise, a amostra foi pré-diluída com $20 \mu \mathrm{L}$ de meio contendo TRIS-Citrato (Quadro1) sem glicerol. Posteriormente, foi avaliado o vigor (em escala de 0 a 5) e a motilidade espermática (percentual de espermatozoides móveis), sob o aumento de 100x ao microscópio de luz monocular portátil (Artificial Insemination Handycope ${ }^{\circledR}$, Freedomand Challenge, Coréia do Sul). Estes valores foram utilizados no cálculo do índice espermático $(\mathrm{IE}=[\mathrm{M}+(\mathrm{Vi} \times 20)] / 2$, em que $\mathrm{M}$ representa a motilidade e Vi o vigor espermático) que consiste na média entre vigor e motilidade espermática na qual ambos têm a mesma significância (Howard et al. 1993).

Para a determinação da concentração espermática do ejaculado utilizou-se uma lâmina especial, contendo uma câmara de $10^{-6}$ $\mathrm{mL}$, fornecida pelo fabricante do microscópio de luz monocular portátil (Artificial Insemination Handycope ${ }^{\circledR}$, Freedomand Challenge, Coréia do Sul). A concentração foi aferida por contagem direta do número de espermatozoides presentes no interior da câmara de contagem, sendo a concentração final expressa em milhões por $\mathrm{mL}$.

Após a pré-diluição em meio sem glicerol e avaliação espermática, a amostra de sêmen foi dividida em duas alíquotas de volume igual e cada uma delas foi rediluída em meio contendo 10 e $15 \%$ de glicerol, na proporção 1:1, de forma a se obter soluções finais com 5 e 7,5\% de glicerol, respectivamente. As amostras foram envasadas em palhetas de $0,25 \mathrm{~mL}$. Para se obter uma taxa de resfriamento de $-0,55^{\circ} \mathrm{C} / \mathrm{min}$ utilizou-se um recipiente térmico de poliestireno expandido com volume de $12 \mathrm{~L}$ contendo mistura de água em temperatura ambiente e gelo, ocupando uma altura de $11 \mathrm{~cm}$ (Bueno et al. 2001 modificado). As palhetas foram colocadas em tubo de ensaio de vidro com tampa rosqueada e este foi imerso em um frasco de vidro com tampa contendo $600 \mathrm{ml}$ de água a $37^{\circ} \mathrm{C}$. Este conjunto foi colocado no recipiente térmico onde permaneceu por duas horas (uma hora de resfriamento e mais uma hora de equilíbrio).

Para o congelamento utilizou-se outro recipiente térmico de poliestireno expandido, sem marca definida, com volume de 12 L contendo nitrogênio liquido a uma altura de aproximadamente três centímetros. Um artefato de poliestireno expandido, no formato de uma pequena caixa sem fundo ou tampa, foi utilizado como boia de forma a manter as palhetas posicionadas horizontalmente a uma altura de $10 \mathrm{~cm}$ da lâmina de nitrogênio líquido e assim congeladas no vapor do nitrogênio. Desta forma obteve-se uma taxa de congelamento de $-5,8^{\circ} \mathrm{C} / \mathrm{min}$. Passados 15 minutos as palhetas foram imersas diretamente no nitrogênio líquido e depois armazenadas em botijão apropriado, para posterior avaliação.

Após uma semana as palhetas foram descongeladas por imersão em água a $37^{\circ} \mathrm{C}$ por 30 segundos. Em seguida o sêmen foi transferido para frascos plásticos de 1,5 mL (Eppendorf), mantidos em banho-maria a $37^{\circ} \mathrm{C}$. Posteriormente, foram analisados 
o vigor e a motilidade espermática como descrito para o sêmen a fresco e conduzidos os testes hiposmótico e de termorresistência.

Uma alíquota de $20 \mu \mathrm{L}$ do sêmen descongelado foi adicionada a $0,5 \mathrm{~mL}$ de formol salino tamponado e 200 células foram avaliadas, ao microscópio de contrate de fase sob o aumento de 1000x, quanto à proporção de espermatozoides normais e com presença de alterações morfológicas. Para realização do teste hiposmótico, uma amostra de $20 \mu \mathrm{L}$ de sêmen foi incubada a $37^{\circ} \mathrm{C}$ por meia hora, em 0,5mL de solução de frutose e citrato de sódio 60 mOsmol/kg (Kumi-Diaka 1993). Em seguida, 200 células foram observadas em microscopia de luz sob o aumento de 1000 vezes para contabilização do percentual bruto de espermatozoides reativos ao teste hiposmótico (espermatozoides com dobramento ou enrolamento de cauda). 0 valor bruto de espermatozoides reativos foi corrigido excluindo-se desta população a parcela com dobramento ou enrolamento de cauda contabilizada no teste de morfologia espermática.

A longevidade espermática foi estimada pelo teste de termorresistência. Para tanto, o sêmen foi incubado em banho-maria a $37^{\circ} \mathrm{C}$ por duas horas, sendo que neste período o vigor e a motilidade espermática foram avaliados em intervalos de 20 minutos. Os dados de vigor e motilidade espermática obtidos durante o teste de termorresistência são apresentados na forma de índice espermático.

Para a análise estatística, a variável qualitativa "vigor" foi submetida ao teste não paramétrico de Kruskal-Wallis. As variáveis quantitativas foram submetidas aos testes de Normalidade (Lilliefors) e Homocedasticidade (Cochran) e quando atenderam às premissas foram submetidos ao teste de comparação de médias de Ducan, adotando-se o nível de 5\% de probabilidade (SAEG 1999).

\section{RESULTADOS}

O procedimento de coleta de sêmen, por eletroejaculação, foi realizado uma vez em cada animal. Apenas o animal 3 apresentou baixa qualidade espermática (vigor 1, motilidade $10 \%$ e concentração $5 \times 10^{6}$ espermatozoides $/ \mathrm{mL}$ ) em relação aos demais animais (Quadro 2), portanto a amostra coletada não foi congelada. Os demais ejaculados (Suçua-

Quadro 1. Componentes utilizados no meio para criopreservação de sêmen de suçuarana

\begin{tabular}{lc}
\hline \multicolumn{1}{c}{ Componentes } & Quantidade \\
\hline TRIS (g) & 3,025 \\
Ácido cítrico (g) & 1,70 \\
Frutose (g) & 1,25 \\
Gema de ovo \% & 20 \\
Estreptomicina (mg/L) & 1 \\
Água destilada q.s.p. & $100 \mathrm{~mL}$
\end{tabular}

Quadro 2. Média do volume ( $\mathrm{mL})$, concentração espermática (espermatozoides $\times 10^{6} / \mathrm{ml}$ ), motilidade espermática (\%), vigor e índice espermático (\%) do sêmen a fresco de machos adultos de suçuarana (S) mantidos em cativeiro

\begin{tabular}{lcccccc}
\hline & S 1 & S 2 & S 3 & S 4 & S 5 & Média \\
\hline Volume & 0,4 & 0,5 & 0,4 & 0,5 & 0,4 & 0,4 \\
Concentração espermática & 180 & 400 & 5 & 60 & 180 & 165 \\
Motilidade espermática & 60 & 70 & 10 & 90 & 80 & 62 \\
Vigor & 3 & 3 & 1 & 4 & 4 & 3 \\
Índice espermático & 60 & 65 & 15 & 85 & 80 & 61
\end{tabular}

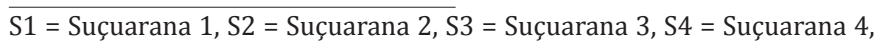
S5 = Suçuarana 5 .
Quadro 3. Médias e Desvio Padrão da motilidade espermática de sêmen fresco e descongelado, em meios com 5,0 e 7,5\% de glicerol, coletados de machos adultos de suçuarana (S) mantidos em cativeiro

\begin{tabular}{lcccccc}
\hline & S 1 & S 2 S & S S 5 & $\begin{array}{c}\text { Médias + Desvio Padrão } \\
\text { (Coeficiente de Variação) }\end{array}$ \\
\hline $\begin{array}{l}\text { Sêmen fresco } \\
\begin{array}{l}\text { Sêmen descongelado, meio } \\
\text { com 5\% de glicerol }\end{array}\end{array}$ & 40 & 40 & 90 & 35 & 50 & $75 \pm 12.91 \mathrm{a}^{*}(17,21)$ \\
$\begin{array}{c}\text { Sêmen descongelado, meio } \\
\text { com 7,5\% de glicerol }\end{array}$ & 40 & 40 & 30 & 50 & $40 \pm 8,16 \mathrm{~b}^{*}(20,40)$ \\
\end{tabular}

* Médias com letras diferentes sobrescritas na mesma coluna são diferentes $(\mathrm{p}<0,05)$ pelo teste de Ducan. S1 = Suçuarana 1, S2 = Suçuarana 2, S4 = Suçuarana 4, S5 = Suçuarana 5.

Quadro 4. Médias \pm Desvio Padrão (Coeficiente de variação) do vigor espermático antes e após o congelamento, em meios com 5 e 7,5\% de glicerol, de sêmen de machos adultos de suçuarana (S) mantidos em cativeiro

\begin{tabular}{|c|c|c|c|c|c|}
\hline & $\mathrm{S} 1$ & S 2 & S 4 & S 5 & $\begin{array}{c}\text { Média + Desvio Padrão } \\
\text { (Coeficiente de Variação) }\end{array}$ \\
\hline Sêmen fresco & 3 & 3 & 4 & 4 & $3,50 \pm 0,58 a^{*}(16,50)$ \\
\hline $\begin{array}{l}\text { Sêmen pós-descongelamento } \\
\text { meio com } 5 \% \text { de glicerol }\end{array}$ & 2 & 3 & 2 & 3 & $2,50 \pm 0,58 a^{*}(23,09)$ \\
\hline $\begin{array}{l}\text { Sêmen pós-descongelamento } \\
\text { meio com } 7,5 \% \text { de glicerol }\end{array}$ & 2 & 2 & 2 & 3 & $2,25 \pm 0,58 a^{*}(22,22)$ \\
\hline
\end{tabular}

* Não houve diferença ( $\mathrm{p}>0.05)$ entre tratamentos, pelo teste não paramétrico de Kruskal-Wallis.

S1 = Suçuarana 1, S2 = Suçuarana 2, S4 =- Suçuarana 4, S5 = Suçuarana 5.

ranas 1, 2, 4 e 5) apresentaram vigor de 3 a 4, motilidade espermática entre 60 e $90 \%$, e concentração entre 60 e $400 \times 10^{6}$ espermatozoides/mL, sendo considerados dentro da média para a espécie (Wildt et al. 1988, Miller et al. 1990).

O sêmen das suçuaranas apresentou motilidade espermática média de 75\%, no sêmen fresco e após o descongelamento a motilidade variou de 35 a $50 \%$ nas amostras diluídas em meio com 5\% de glicerol e de 30 a $50 \%$ em meio com 7,5\% de glicerol (Quadro 3).

0 vigor espermático do sêmen fresco de suçuarana avaliado no presente trabalho apresentou valor médio de 3,5 e nas amostras descongeladas variaram de 2 a 3 nas concentrações de 5 e 7,5\% de glicerol, respectivamente (Quadro 4).

Os índices espermáticos de amostras frescas de sêmen de suçuarana apresentaram média de 72,5\%, enquanto as amostras descongeladas apresentaram índices espermáticos entre 37,5 e $52,5 \%$ no meio com $5 \%$ de glicerol e 35 a $55 \%$ no meio contendo 7,5\% de glicerol (Quadro 5).

Foram observados espermatozoides vivos até 60 minutos após o descongelamento nas amostras das Suçuaranas 2 e 5, em ambas as concentrações de glicerol, a amostra menos termorresistente foi congelada com 7,5\% de glicerol, da Suçuarana 1 (Quadro 6).

0 percentual de espermatozoides que se apresentaram com membrana funcional após o descongelamento, de acordo com o Teste Hiposmótico, variou de 8\% (Suçuarana 3 com 7,5\% de glicerol) a 52\% na amostra da Suçuarana 4 congelada em meio com $5 \%$ de glicerol (Quadro 7). 
Quadro 5. Médias e Desvio Padrão do índice espermático antes e após o congelamento, em meios com 5 e $7.5 \%$ de glicerol, de sêmen de machos adultos de suçuarana (S) mantidos em cativeiro

\begin{tabular}{lcccccc}
\hline & S1 & S 2 & S 4 & S 5 & $\begin{array}{c}\text { Média + Desvio Padrão } \\
\text { (Coeficiente de Variação) }\end{array}$ \\
\hline $\begin{array}{l}\text { Sêmen fresco } \\
\begin{array}{l}\text { Sêmen após o descon- } \\
\text { gelamento, meio com }\end{array}\end{array}$ & 40 & 65 & 52.5 & 37.5 & 55 & $46,50 \pm 11,90 a^{*}(16,42)$ \\
$\quad \begin{array}{l}5 \% \text { de glicerol } \\
\text { Sêmen após o descon- } \\
\text { gelamento, meio com }\end{array}$ & 40 & 40 & 35 & 55 & $42,50 \pm 8,66 b^{*}(20,38)$ \\
$\quad 7,5 \%$ de glicerol & & & & & & \\
\hline
\end{tabular}

* Médias com letras diferentes sobrescritas na mesma coluna são diferentes $(\mathrm{p}<0.05)$ pelo teste de Ducan. S1 = Suçuarana 1, S2 = Suçuarana 2. S4 =Suçuarana 4. S5 = Suçuarana 5 .

Quadro 6. Médias e Desvio Padrão do índice espermático do sêmen descongelado e incubado a $37^{\circ} \mathrm{C}$, de machos adultos de suçuarana (S) mantidos em cativeiro

\begin{tabular}{|c|c|c|c|c|c|}
\hline $\begin{array}{l}\text { Tempo de incubação } \\
\text { e meio avaliado }\end{array}$ & S 1 & S 2 & S 4 & S 5 & $\begin{array}{c}\text { Média + Desvio } \\
\text { Padrão (Coeficiente } \\
\text { de Variação) }\end{array}$ \\
\hline $\begin{array}{l}\text { T } 0 \text { min } \\
\text { Meio com } 5 \% \text { de glicerol }\end{array}$ & 40 & 52,50 & 37,50 & 55 & $\begin{array}{c}46,25 \pm 8,78 a^{*} \\
(18,98)\end{array}$ \\
\hline $\begin{array}{l}\text { T 0min } \\
\text { Meio com } 7.5 \% \text { de glicerol }\end{array}$ & 40 & 40 & 35 & 55 & $\begin{array}{c}42,50 \pm 8,66 \mathrm{a}^{*} \\
(20,38)\end{array}$ \\
\hline $\begin{array}{l}\text { T 20min } \\
\text { Meio com } 5 \% \text { de glicerol }\end{array}$ & 20 & 40 & 20 & 35 & $\begin{array}{c}28,75 \pm 10,31 b^{*} \\
(35,85)\end{array}$ \\
\hline $\begin{array}{l}\text { T } 20 \mathrm{~min} \\
\text { Meio com } 7,5 \% \text { de glicerol }\end{array}$ & 17,50 & 40 & 17,50 & 35 & $\begin{array}{c}27,50 \pm 11,73 \mathrm{~b}^{*} \\
(42,64)\end{array}$ \\
\hline $\begin{array}{l}\text { T } 40 \mathrm{~min} \\
\text { Meio com } 5 \% \text { de glicerol }\end{array}$ & 12,50 & 20 & 15 & 17,50 & $\begin{array}{c}16,25 \pm 3,23 c^{*} \\
(19,86)\end{array}$ \\
\hline $\begin{array}{l}\text { T } 40 \mathrm{~min} \\
\text { Meio com } 7,5 \% \text { de glicerol }\end{array}$ & 0 & 17,50 & 12,50 & 15 & $\begin{array}{c}11,25 \pm 7,77 c, d^{*} \\
(69,09)\end{array}$ \\
\hline $\begin{array}{l}\text { T } 60 \mathrm{~min} \\
\text { Meio com } 5 \% \text { de glicerol }\end{array}$ & 0 & 12,50 & 0 & 12,50 & $\begin{array}{c}6,25 \pm 7,22 c, d^{*} \\
(115,47)\end{array}$ \\
\hline $\begin{array}{l}\text { T } 60 \mathrm{~min} \\
\text { Meio com } 7,5 \% \text { de glicerol }\end{array}$ & 0 & 12,50 & 0 & 12,50 & $\begin{array}{c}6,25 \pm 7,22 c, d^{*} \\
(115,47)\end{array}$ \\
\hline $\begin{array}{l}\text { T } 80 \mathrm{~min} \\
\text { Meio com } 5 \% \text { de glicerol }\end{array}$ & 0 & 0 & 0 & 0 & $0 \pm 0,00 \mathrm{~d}^{*}(0)$ \\
\hline $\begin{array}{l}\text { T } 80 \mathrm{~min} \\
\text { Meio com } 7,5 \% \text { de glicerol }\end{array}$ & $0^{\mathrm{c}}$ & $0^{\mathrm{c}}$ & $0^{c}$ & $0^{c}$ & $0 \pm 0,00 \mathrm{~d}^{*}(0)$ \\
\hline
\end{tabular}

Quadro 7. Média e Desvio Padrão do teste hiposmótico após o descongelamento, em meios com 5 e 7.5\% de glicerol, de sêmen de machos adultos de suçuarana (S) mantidos em cativeiro

Meio avaliado S 1 S 2 S 4 S 5 Média + Desvio Padrão (Coeficiente de Variação)

Meio com 5\% de glicerol (\%)** 21321252 29,25 \pm 17,23a* (58,91) Meio com 7,5\% de glicerol (\%)** $24 \quad 22 \quad 8 \quad 48$ 25,50 \pm 16,60a* $(65,11)$

* Médias com letras diferentes sobrescritas na mesma coluna são diferentes $(\mathrm{p}<0.05)$ pelo teste de Ducan. ${ }^{* *}$ Percentual de espermatozoides com cauda enrolada após teste hiposmótico reduzido do percentual de espermatozoides com cauda enrolada patológica. S1 = Suçuarana 1, S2 = Suçuarana 2, S4 = Suçuarana 4, S5 = Suçuarana 5.

\section{DISCUSSÃO}

O glicerol é um crioprotetor extremamente eficiente, cuja toxidade é influenciada pela concentração usada, tempera- tura a qual foi adicionado à amostra e da espécie estudada (Fahy 1986, England 1993, Nelson et al. 1999, Holt 2000, Santos et al. 2003, Pesch \& Bergmann 2006). Stander-Breedtet al. (2004) testaram o efeito do glicerol e do DMSO nas concentrações 4 e $8 \%$ para congelamento de sêmen em leão (Panthera leo), e não observaram diferenças significantes na motilidade espermática, após o descongelamento entre os quatro tratamentos. Da mesma forma, a presente experimentação não observou diferença $(p>0,05)$, quanto à motilidade espermática, entre as amostras congeladas com 5 e $7,5 \%$ de glicerol.

A motilidade espermática após o descongelamento observada no presente experimento variou de 40 a 42,5\%, representando uma queda média de 33,75\% em relação ao sêmen fresco. Um estudo com humanos observou correlação entre a motilidade espermática e a fertilidade. Sêmen fresco apresentando motilidade espermática $>50 \%$ no momento da fertilização apresentou taxas de $75,8 \%$ de fertilização enquanto que aqueles com motilidade entre $30 \mathrm{a}$ $49 \%$ apresentaram fertilidade de $49,1 \%$ e aqueles com motilidade $<20 \%$ apresentaram completa falha na fertilização (Mahadevan \& Trounson 1984). Sabidamente o processo de criopreservação provoca danos aos espermatozoides reduzindo seus parâmetros de motilidade e vigor e, consequentemente, a capacidade de fertilização. Baixos valores de motilidade espermática, no entanto, podem ser compensados depositando o sêmen mais próximo do local da fertilização, aumentando assim a taxa de fertilidade (Villaverde \& Lopes 2007).

0 índice espermático avaliado imediatamente após o descongelamento $(42,5$ a $46,25 \%)$ do sêmen de suçuaranas declinou $(p<0,05)$ em relação ao sêmen fresco $(72,5 \%)$, porém não houve diferença ( $p>0,05)$. Em estudos com animais silvestres, em especial os felídeos, análises de correlação da fertilidade com os diversos parâmetros de avaliação in vitro são pouco precisas, dado à reduzida quantidade de amostras a serem avaliadas, visto a dificuldade de acesso a esses animais e ao pequeno volume obtido no ejaculado.

A funcionalidade da membrana é essencial para que ocorra a fertilização sendo, portanto um importante parâmetro seminal que pode ser avaliado pelo teste hiposmótico (HOST). Em humanos, amostras de sêmen com valores baixos para este teste $(<50 \%)$ formam embriões com baixa capacidade de implantação (Checket al. 1995, Katsoffet al. 2000). Segundo Check et al. (2001), uma possível explicação é que a alteração na membrana demonstrada no teste está relacionada com um fator tóxico (possivelmente uma proteína) presente na membrana plasmática do espermatozoide que pode ser transferido ao embrião e impedir que este se implante no útero. Em garanhões observou-se correlação entre o HOST e a motilidade espermática e a taxa de prenhez (Neildet al. 2000). No presente experimento, não houve diferença para esse teste entre as duas concentrações de glicerol usadas $(p>0,05)$. Semelhantemente, em cães a porcentagem de espermatozoides reativos ao HOST não variou em sêmen congelado com 4 e $6 \%$ de glicerol (média de 18,61\% para ambas) (Mascarenhas 2008), porém os valores se apresentaram muito abaixo aos encontrados em suçuaranas neste experimento. 
0 teste de termorresistência avalia a longevidade espermática e é tido como um bom preditor da fertilidade em várias espécies (England 1993). A longevidade espermática se mostrou equivalente $(p>0,05)$ para ambas as concentrações de glicerol utilizadas neste experimento. Ainda de acordo com este teste, o sêmen de suçuaranas, criopreservado com 5 e 7,5\% de glicerol, apresentou índice espermático médio de 28,75 e $27,5 \%$, respectivamente, valores próximos aos encontrados em sêmen de cães após 30 minutos de incubação $(25,89 \%)$ (Mascarenhas 2008).

A qualidade espermática após o descongelamento é afetada pelas várias etapas da criopreservação, desde o envasamento e concentração do crioprotetor utilizado até as taxas de resfriamento, congelamento e descongelamento. A influência destas etapas se dá de forma sinérgica, logo para se determinar o protocolo de criopreservação ideal para cada espécie faz-se necessária a análise conjunta destas variáveis. No entanto, o avanço no conhecimento sobre a congelabilidade de sêmen de espécies não domésticas, especialmente os grandes felídeos, esbarra na dificuldade de obtenção de amostras significativas, dado a raridade de espécimes e a complexidade no manejo destes animais. Sendo assim, dado a grande gama de técnicas a serem testadas ainda não se descreveu um protocolo definitivo, porém, de cada experimentação soma-se parâmetros e procedimentos neste sentido.

\section{CONCLUSÕES}

0 protocolo proposto para coleta de sêmen de suçuarana mantidos em cativeiro mostrou-se eficiente com a obtenção de amostras livres de contaminação com urina e em concentração satisfatória.

Os valores do índice espermático no teste de termorresistência assim como o teste hiposmótico demonstraram que ambas as concentrações de glicerol utilizadas no presente experimento apresentam potencial de uso eficaz na criopreservação de sêmen de suçuaranas adultos mantidos em condições de cativeiro, uma vez que apresentaram resultados satisfatórios nos parâmetros testados.

Ao teste de termorresistência, o sêmen de suçuaranas apresentou índice espermático em patamares considerados viáveis para inseminação pelo menos 20 minutos depois de descongelado.

\section{REFERÊNCIAS}

Bueno R., Costa E.P., Guimaräes J.D. \& Valentim F.M. 2001. Qualidade espermática do sêmen criopreservado de cães: efeito do meio diluidor. Arq. Bras. Med. Vet. Zootec. 53:364-371.

Check J.H., Katsoff D. \& Check M.L. 2001. Some semen abnormalities may cause infertility by impairing implantation rather than fertilization. Med. Hypotheses 56:653-657.

Check J.H., Stumpo L., Lurie D., Benfer D.K. \& Callan C. 1995. Fertilization and early embryology: A comparative prospective study using matched samples to determine the influence of subnormal hypoosmotic test scores of spermatozoa on subsequent fertilization and pregnancy rates following in vitro fertilization. Human Reprod. 10:1197-1200.
Deco T.S., Paula T.A.R., Costa D.S., Araújo G.R., Garay R.M., Vasconcelos G.S.C., Csermak Junior A.C., Carretta-Júnior M., Silva L.C. \& Barros J.B.G. 2010. Coleta e avaliação de sêmen de puma (Puma concolor Linnaeus, 1771) adultos mantidos em cativeiro. Revta Bras. Reprod. Anim. 34: 252-259.

England G.C. 1993. Cryopreservation of dog semen: A review. J. Reprod. Fertility 47(Suppl.1):234-255.

Fahy G.M. 1986. The relevance of cryoprotectant "toxicity" to cryobiology. Cryobiol. 23:1-13.

Holt W.V. 2000. Basic aspects of frozen storage of semen. Anim. Reprod. Sci. 62:3-22.

Howard J.G., Donoghue A.M., Johnston L.A. \& Wildt D.E. 1993. Zona pellucida filtration of structurally abnormal spermatozoa and reduced fertilization in teratospermic cats. Biol. Reprod. 49:131-139.

Katsoff D., Check M.L. \& Check J.H. 2000. Evidence that sperm with low hypoosmotic swelling scores cause embryo implantation defects. Arch. Androl. 44:227-230.

Kumi-Diaka J. 1993. Subjecting canine semen to the hypo-osmotic test. Theriogenology 39:1279-1289.

Mahadevan M.M. \& Trounson A.O. 1984. The influence of seminal characteristics on the success rate of human in vitro fertilization. Fertil. Steril. 42:400-405

Mascarenhas R.M. 2008. Padrão racial do sêmen canino fresco e as variações em sua congelabilidade entre diferentes raças e protocolos de congelamento. Dissertação de Mestrado, Universidade Federal de Viçosa, Viçosa, MG. 83p.

Miller A.M., Roelke M.E., Goodrowe K.L., Howard J.G. \& Wildt D.E. 1990. Oocyte recovery, maturation and fertilization in vitro in the puma (Felis concolor). J. Reprod. Fertil. 88:249-258.

Neild D.M., Chaves M.G., Flores M., Miragaya M.H., Gonzalez E. \& Agüero A. 2000. The HOS test and its relationship to fertility in the stallion. Androl. 32:351-355.

Nelson K.L., Crichton E.G., Doty L., Volenec D.E., Morato R.G., Pope C.E., Dresser B.L., Brown C.S., Armstrong D.L. \& Loskutoff N.M. 1999. Heterologous and homologous fertilizing capacity of cryopreserved felid sperm: A model for endangered species. Theriogenology 51:290. (Abstract)

Pesch S. \& Bergmenn M. 2006. Structure of mammalian spermatozoa in respect to viability, fertility and cryopreservation. Micron 37:597-612.

Santos I.W., Lima V.F.M.H., Nisfeld L.C. \& Ribeiro A.P.C. 2003. Congelação do sêmen canino comparando a diferentes concentrações de glicerol e diferentes tempos de equilíbrio. Arch. Vet. Sci. 8:57-62.

SAEG 1999. Central de Processamento de Dados. Sistema de Análise Estatística e Genética, Viçosa, MG.

Stander-Breedt H., Schwalbach L.M.J., Geyling J.P.C. \& Loskutoff N.M. 2004. Effect of different cryodiluents and thawing methods on the post-thaw motility of African lion (Panthera leo) spermatozoa. South African J. Anim. Sci. 34:74-76.

Swanson W.F. \& Brown J.L. 2004. International training programs in reproductive sciences for conservation of Latin American felids. Anim. Reprod. Sci. 82/83:21-34.

Villaverde A.I.S.B. \& Lopes M.D. 2007. Inseminação artificial em gatos domésticos utilizando sêmen criopreservado. Revta Bras. Reprod. Anim. 31:77-83.

Wildt D.E., Phillips L.G., Simmons L.G., Chakraborty P.K., Brown J.L., Howard J.G., Teare A., Bush M. 1988. A comparative analysis of ejaculate and hormonal characteristics of the captive male cheetah, tiger, leopard, and puma. Biol. Reprod. 38:245-255.

Zambelli D., Merlo B., Iacono E., Prati F. \& Belluzzi S. 2006. Fertilizing ability of electro-ejaculated cryopreserved semen in the domestic cat. Reprod. Dom. Anim. 41:137-141. 